\title{
Unilateral Unascended Kidney with Vascular Anomalies: A Cadaveric Case Report
}

\author{
Authors \\ Dr Sushil Kumar*, Dr Debasis Bandopadhyay** \\ *Prof \& Head, Dept of Anatomy, AFMC, Pune - 411040. \\ **Associate Professor, Dept of Anatomy, AFMC, Pune - 411040 \\ Dept of Anatomy, Armed Forces Medical College, Pune-411040 \\ Email: debasis13@hotmail.com
}

\begin{abstract}
The anomalies of kidney form a sizeable part of all the congenital malformations. Generally defects in ascent of kidney are associated with variations in branching pattern of the abdominal aorta. Here we report a case of unilateral unascended right kidney with malrotation in a 60 yrs old male cadaver with associated vascular variations. The right kidney was larger in size, laterally rotated and placed at a lower lumbar level showing varied branching pattern of renal vasculature. The left kidney was normally placed with hilum directed medially and a normal renal vasculature.

Failure of the kidney to ascend into the renal fossa in utero results in Ectopic kidney. Such kidney is often found in the pelvis however it may be placed higher up in lower lumbar region. Pelvic kidneys often become hydronephrotic due to obstruction of the anteriorly placed ureter and an anomalous arterial supply. No such gross changes were seen in our case.

The embryological basis of rotational anomalies is still unknown. Rotational anomaly though rare, has important implications from surgical point of view, as it may be mistaken for some more serious condition on Intravenous Pyelography (IVP). It may erroneously be attributed to displacement by para vertebral mass / tumour. It also assumes a great importance in the context of present day surgical procedures like percutaneous nephrectomy where modified technique is required for upper pole access in malrotated kidneys. Though malrotated kidney without any history of calculi or hydronephrosis is hardly rejected for donation a detailed CT Scan is a must for preoperative diagnostic evaluation of donors to minimise iatrogenic damages. Keywords: Ectopic kidney, Malrotation, hilum, hydronephrotic, pyelography, nephrectomy
\end{abstract}

\section{Introduction}

Normally the kidneys are retroperitoneal lying in the upper parts of paravertebral gutters approximately $10 \mathrm{~cm}$ long, $5 \mathrm{~cm}$ wide and $2.5 \mathrm{~cm}$ thick extending between transverse processes of
T12 to L3 vertebrae with the left kidney typically somewhat more superior in position than the right. The upper poles are normally oriented more medially and posteriorly than the lower poles. ${ }^{1}$ Kidneys are prone to embryological 
malformations in terms of number, position, shape, size, rotation and vascularisation. Congenital anomalies of the kidney and urinary tract anatomy are common entity in children and represent approximately $30 \%$ of all prenatally diagnosed malformations. ${ }^{2}$

The kidneys develop from intermediate mesoderm forming nephrogenic cord in the fourth week of intrauterine life along the posterior wall of the abdominal cavity. Three overlapping kidney systems are formed in a cranio-caudal sequence called pronephros, mesonephros and metanephros. The pronephros represented in the cervical region completely regresses, the mesonephros representted in the thoracolumbar region acts as interim kidney between $4^{\text {th }}$ to $8^{\text {th }}$ week of intrauterine life and then regresses while metanephros represented in the sacral region forms the definitive kidney. ${ }^{3}$ At first the permanent kidney derived from the metanephros lies in the sacral region. Later due to differential growth of posterior abdominal wall and reduction in the flexed curvature of the fetus the kidneys ascend to the thoraco-lumbar region. In the pelvis the metanephros receives its arterial supply from pelvic branch of aorta. During its ascent to the abdominal level a series of vessels form to supply it only to involute again till supply from renal artery is established. Some of these fetal renal arteries do not degenerate and persists in adult as aberrant / accessory renal arteries. During ascent the kidneys pass through the arterial fork formed by the umbilical arteries and one of them may fail to ascend because of the obstruction of sickle shaped fold of peritoneum projecting from the lateral pelvic wall containing the umbilical artery. Such kidney remaining in the Pelvis close to the common iliac artery is known as the Pelvic kidney. Ectopic kidney is described as abnormal localization of a kidney due to a developmental anomaly occurring as a result of a premature halt in migration of the kidney to its normal location during the embryonic period. An ectopic kidney, more frequent in males, can be found in the pelvis, abdomen or even thorax. There is a strong correlation between renal ascension and vascularization. Any presence of aberrant arteries can stop the ascent and cause ectopia or rotation anomalies. During ascent, the hilum of the kidney is directed ventrally. On reaching the definitive position it undergoes $90^{\circ}$ medial rotation around the vertical axis such that the hilum directs medially. ${ }^{4}$ The exact cause of malrotation of the kidneys is not known but it is believed that malrotation occurs due to abnormal insertion of the ureteric bud into an abnormal region of metanephros. When associated with renal ectopia malrotation probably occurs due to incomplete medial rotation brought about by the aberrant vessels.

Unilateral renal ectopia is most frequent with incidence ranging from 1:1000 to 1:300 births. ${ }^{5,6}$ Generally, an ectopic kidney is smaller, of irregular shape and variable rotation and is vascularised by multiple arteries with various levels of origin. Kidneys in ectopic position may be dysplastic and often non-functional. They may go undetected in life and get noticed only after death either in autopsy or during dissection. Often they are diagnosed when investigating for presence of a pelvic mass or on pyelogram. The increase in renal transplants, vascular reconstruction and other urologic procedures requires a detailed knowledge of the renal morphological, positional and vascular variations and hence our case report is an attempt to add to the available data on these parameters.

\section{Case Report}

During routine undergraduate dissection of the abdominal region a right unascended kidney was noticed in a 60 yrs old male cadaver. Though both kidneys had a normal gross appearance the right kidney was relatively lower. The left kidney was normally placed with hilum at lower border of L1 vertebrae. The right kidney extended from lower border of L2 to upper border of S1 with hilum directed ventrally at the level of body of L4. There were multiple renal arteries seen arising from the abdominal aorta and multiple renal veins draining into inferior venacava (Fig 1). 


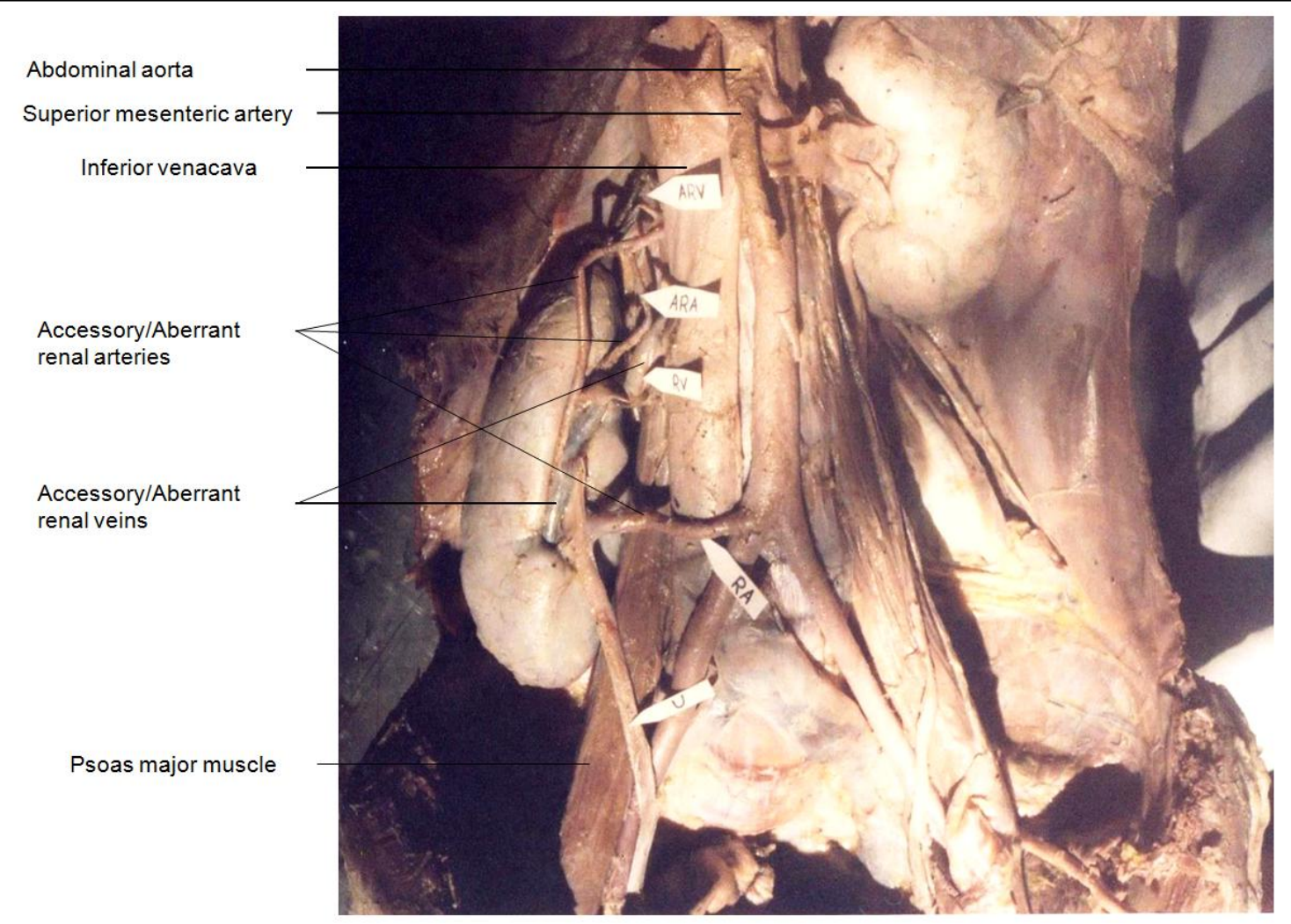

Fig 1: Right unascended kidney with hilum directed ventrally and multiple accessory/aberrant renal vessels. ARV: Aberrant renal vein, ARA: Aberrant renal artery, U : Ureter

\section{Discussion}

The anomalies of kidney form a sizeable part of all the congenital malformations. Initially the primordial permanent kidneys derived from metanephros lies in the pelvis ventral to the sacrum. Due to differential growth of the posterior abdominal wall the kidneys ascend and come to lie in the abdomen by $9^{\text {th }}$ week of intrauterine life. Failure to ascend leads to ectopic kidney which may be located commonly in the pelvis and sometimes in the abdomen. Though arterial fork formed by the umbilical arteries is the commonest reason for unilateral pelvic kidney other factors like effect of teratogenic agents, chromosomal disorders and improper fusion of ureteric bud with metanephric blastema may contribute to incomplete ascent. ${ }^{2,3}$ Very rarely excessive cranial ascent of kidney prior to diaphragmatic closer can lead to a ectopic thoracic kidney. ${ }^{7}$ Generally ectopic kidney is smaller in size but in our case the right kidney was normal in size and gross appearance. It was located lower in the abdomen extending from lower border of L2 to upper border of $\mathrm{S} 1$. It measured $13 \mathrm{cms}$ vertically,
$8.5 \mathrm{cms}$ transversely and $3 \mathrm{cms}$ antero-posteriorly (thickness).

Initially the hilum of the kidney faces ventrally but as it ascends cranially the hilum rotates medially by $90^{\circ}$ around a vertical axis. At $9^{\text {th }}$ week of intrauterine life the hilum faces anteromedially and eventually faces medially after it becomes retroperitoneal. In our case the hilum of the right kidney was ventrally directed. Anomalies of rotation may be seen in normal and ectopic kidney. The rotational anomalies may vary from an anteromedial position of hilum in cases of incomplete rotation to ventrally directed hilum in cases of non rotation. Only in rare cases the hilum is laterally directed due to excessive rotation or reverse rotation. ${ }^{8,9}$ In our case the right kidney was ventrally directed probably due to non rotation.

The fetal renal arteries arise successively in sequence from the aorta as the kidney ascends from pelvic to lumbar region. During embryological period, the metanephros derives its blood supply from median sacral artery which is a pelvic continuation of aorta initially followed by 
common iliac and inferior mesenteric arteries and lastly from aorta. During the ascent, the former vessels degenerate and finally only a pair of renal arteries supply the kidneys at L1-L2 level. Failure of degeneration of these vessels result in origin of accessory renal arteries. ${ }^{3}$ They represent persistent fetal arteries.

There is also a correlation between the kidney ascent and the level of origin of the definitive renal arteries; any anomaly in renal artery development may hold up kidney migration leading to ectopic kidney. The presence of accessory arteries at the apical segment can be explained by a slower than usual process of craniocaudal degeneration and subsequent persistence of mesonephrotic branches. Inferior arteries may develop through a delayed process (in the inferior lumbar region) or ascend with the kidney. ${ }^{3}$ In our case accessory arteries are seen in apical and hilar region of the right kidney suggesting a failure of degeneration of higher lateral splanchnic branches of aorta supplying the kidney and their subsequent persistence in adult life.

Pelvic kidneys often become hydronephrotic due to obstruction of the anteriorly placed ureter and an anomalous arterial supply. However no such gross changes were seen in our case. Rotational anomaly though rare, has important implications from surgical point of view, as it may be mistaken for some more serious condition on Intravenous Pyelography (IVP). It may erroneously be attributed to displacement by para vertebral mass / tumour. It also assumes a great importance in the context of present day surgical procedures like percutaneous nephrectomy where modified technique is required for upper pole access in malrotated kidneys. Though malrotated kidney without any history of calculi or hydronephrosis is hardly rejected for donation a detailed CT Scan is a must for preoperative diagnostic evaluation of donors to minimise iatrogenic damages. Since ectopic kidney is commonly associated with vascular anomalies our case report will add to available data on anatomic variations in the region.

\section{Conclusion}

The ectopic kidney is clinically significant due to its association with vascular anomalies and malrotation. Since it has an atypical location ectopic kidney is more prone to trauma and may also cause hydronephrosis due to obstruction of flow of urine through the ureter most common being at pelvi-ureteric junction. Since intravenous pyelography may erroneously mistaken an ectopic kidney for paravertebral mass computed tomography and magnetic resonance imaging are best diagnostic tools for ectopic kidney. ${ }^{10,11}$

\section{References}

1. Romanes GJ, Cuningham's Manual of Practical Anatomy.Vol. II: Thorax and Abdomen, 15th edition, English Language Book Society, Oxford University Press, 1986.

2. Queisser - Luft A, Stolz G, Wiesel A, et al. Malformations in newborn: results based on 30,940 infants and foetuses from the Mainz congenital birth defects monitoring system (1990-1998). Arch Gynaecol Obstet 2002;266:163.

3. Moore KL, Persaud TVN, The developing human: clinically oriented embryology, 8th edition, Elsevier-WB Saunders, Philadelphia, 2008, 244-256

4. A K Dutta: Essentials Of Human Embryology. $6^{\text {th }}$ edition August 2011. 206207

5. Asghar M, Wazir F, Prevalence of renal ectopia by diagnostic imaging, Gomal $\mathbf{J}$ Med Sci, 2008, 6(2):72-76.

6. Bergman R.A., Afifi A.K. and Miyauchi R In: Illustrated Encyclopedia of human anatomic variation. opus IV: Organ system: Urinary system: Kidneys, ureters, bladders and urethra @ www/virtual hospital.com. 
7. Campbell, M.F Renal ectopy. Journal Of Urolgy, 1930, 24, 187- 189

8. Braash W. F. (1931): Anomalous renal rotation and associated anomalies. Journal of Urology. 25: 9.

9. Ingole IV, Ghosh SK, Laterally rotated kidney - a rare congenital anomaly, J Anat Soc India, 2005, 54(1):19-21.

10. Dretler SP, Olsson C, Pfister RC, The anatomic,radiologic and clinical characteristics of the pelvic kidney:an analysis of 86 cases, J Urol, 1971,105(5):623-627.

11. Asghar M, Wazir F, Prevalence of renal ectopia by diagnostic imaging, Gomal J Med Sci, 2008, 6(2):72-76. 